\title{
Pattern electroretinography in patients with delayed pattern visual evoked potentials due to distal anterior visual pathway dysfunction
}

\author{
GRAHAM E HOLDER \\ From the Regional Department of Clinical Neurophysiology, Brook General Hospital, Shooters Hill Road, \\ London
}

SUMMARY Between March 1983 and January 1988 delayed pattern visual evoked potentials (PVEP) were observed in 67 patients with distal visual pathway dysfunction. Many of these patients had been referred for neurophysiological examination because of possible optic nerve dysfunction. These patients also had pattern electroretinography (PERG) performed which in all cases showed an abnormality of the main positive P50 component. None of these patients had an abnormality confined to the negative N95 component, the type of abnormality usually found if the PERG is abnormal in optic nerve disease. It is suggested that PERG recording should now be a routine adjunct to the PVEP in the assessment of anterior visual pathway dysfunction.

Patients with symptoms of anterior visual pathway dysfunction or of possible multiple sclerosis are frequently referred for pattern visual evoked potential (PVEP) examination. Although optic nerve disease often causes a delayed PVEP, retinal disease can also result in PVEP delays. ${ }^{1-6}$ It is therefore essential that the presence of a PVEP delay is not assumed necessarily to indicate optic nerve dysfunction. This report presents the pattern electroretinogram (PERG) findings in 67 patients with delayed PVEPs due to dysfunction distal to the optic nerve. A preliminary account of this work has been presented.?

\section{Patients and methodology}

The 67 patients were all routinely referred for electrodiagnostic study to the South East Thames Regional Department of Clinical Neurophysiology at the Brook Hospital between March 1983 and January 1988. The final diagnoses of patients with PVEP delays and PERG recording examined during this period were reviewed. This report describes patients with a PVEP delay due to anterior visual pathway dysfunction distal to the optic nerve. The diagnoses in the patients who fulfilled these criteria are shown in table 1.

Correspondence to: Dr G E Holder, Regional Department of Clinical Neurophysiology, Brook General Hospital, Shooters Hill Road, London SE18 4LW, United Kingdom.

Received 2 September 1988 and in revised form 24 April 1989. Accepted 27 June 1989
Many patients referred for retinal assessment do not have VEP recording. It has, however, become routine practice in 0 our laboratory to record the PERG in patients with an abnormal PVEP.

PVEP recording was performed according to standard 8 techniques which have been fully described elsewhere. ${ }^{8}$ Binocular PERG recording was performed using gold foilo corneal electrodes (Carter and Hogg, London) placed in the? fornices of the lower eyelids. Reference electrodes were placed at the ipsilateral outer canthi. ${ }^{10}$ Use of this recording technique ensures that there is no significant contamination either from the fellow eye or from the cortically generated PVEP. ${ }^{11-13}$ Binocular stimulation was delivered by a moving mirror checkerboard stimulator subtending a total field of

Table 1 Diagnoses in 67 patients with PVEP delays

Degenerative*

Retinal vascular lesion

Inflammatory

Amblyopia

Media opacity

Stargardt's disease

Refractive error

Retinal detachment

Diabetic retinopathy

Disciform macular degeneration

Glaucoma

Placoid

Quinine retinopathy

Central serous retinopathy

Hypothyroidism

Undiagnosed maculopathy

Total

*Includes retinitis pigmentosa type conditions. 
Table 2 PERG normal values (16 subjects, 18-48 years)

\begin{tabular}{|c|c|c|c|c|c|c|c|c|}
\hline & \multicolumn{2}{|c|}{ Absolute latency } & \multicolumn{3}{|c|}{ Absolute amplitude (peak-to-peak) } & \multicolumn{3}{|c|}{ Interocular amplitude ratio } \\
\hline & P50 & N95 & P50 & N95 & $N 95: P 50$ & P50 & N95 & N95:P50 \\
\hline
\end{tabular}

$14^{\circ}$ with an individual check subtense of $34^{\prime}$. Stimulus intensity was $400 \mathrm{~cd} / \mathrm{m}^{2}$ with a contrast of $89 \%$. Signal processing was performed by a Nicolet CA1000 averager with a bandwidth of $1-100 \mathrm{~Hz}$. At least two trials consisting of a minimum of 200 averages were acquired using the "interrupted stimulation" technique which has previously been reported to minimise inter-trial variability. ${ }^{13}$ The patient is instructed not to blink while the pattern is reversing, but simply to look at the fixation spot in the centre of the screen and keep still. Every four or five seconds the movement of the pattern is suspended, data collection is stopped and the patient told to blink a few times. The patient is then instructed to resume fixation and averaging is resumed when the input signal has stabilised. This technique is used in conjunction with the internal artefact reject system of the averager. Full details of electrode position, and other technical considerations during recording have appeared elsewhere. $^{14}$ The application of high technical standards is necessary to ensure clinically reliable data.

\section{Results}

The normal PERG using the techniques described consists of a main positive P50 component with a mean latency of some $52 \mathrm{~ms}$ followed by a larger negative N95 component at some $93 \mathrm{~ms}$. Normal values are described in full elsewhere, ${ }^{13}$ and are summarised in table 2 . In many patients these are preceded by a small negative component at some $35 \mathrm{~ms}$ (N35). PERG analysis concentrated on the latency of P50 and the amplitudes (peak-to-peak) of

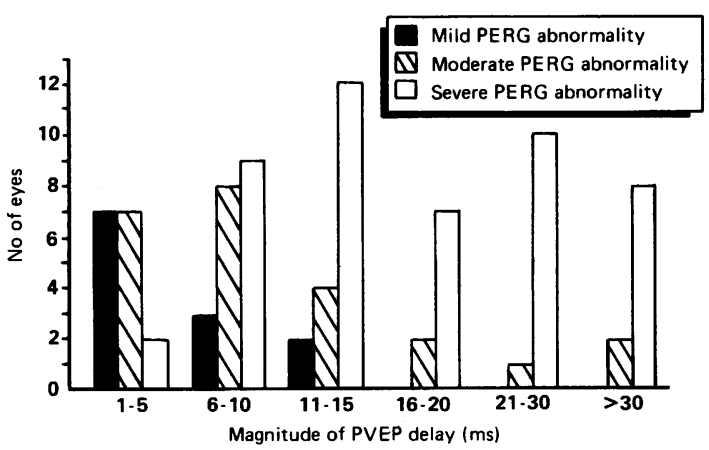

Fig 1 Histogram detailing the PERG and PVEP findings in 84 eyes with distally generated PVEP delays. PVEP delays are given in msec above the upper limit of normal for age. See text for details of PERG abnormality classification.
P50 and N95. In our experience use of the "interrupted stimulation" technique is essential to reduce N95 variability to a minimum, but some authors ${ }^{6}$ with particularly sophisticated data acquisition/artefact reject systems do not find that this technique offers any particular advantages. PVEP analysis was purely concerned with the latency of the major positive component (P100).

All 67 patients had PVEP P100 component latency delays in one or both eyes, which was a criterion for inclusion in the study. The PVEP delay was unilateral in 50 patients, bilateral in 17 patients. The PVEP latency delays in the 84 eyes are shown in fig 1 . Typical findings are shown in figs 2-5.

The P50 component of the PERG was abnormal in all eyes with PVEP delays. The abnormalities in fig 1 appear as mild, moderate and severe. A mild abnormality was defined as an eye with an absolute P50 component amplitude of 1.5-1.9 $\mu \mathrm{V}$ or an interocular P50 amplitude ratio of $0 \cdot 51-0 \cdot 70$. A moderate abnormality was defined as an absolute P50 amplitude of $1.0-1.4 \mu \mathrm{V}$ or an interocular P50 ratio of $0.30-0.50$. Only one patient (with a retinal branch vein occlusion) had a marked P50 latency delay with no associated amplitude changes. This has also been classified as moderate. A severe abnormality was an absolute

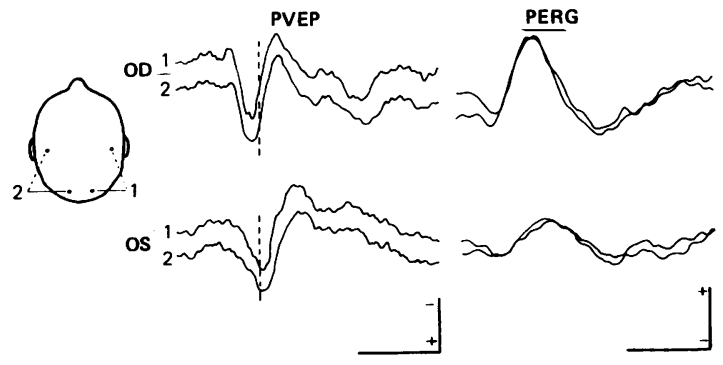

Fig 2 A 39 year old male with a six week history of blurred vision in his left eye. He was referred for VEP examination with a provisional diagnosis of left retrobulbar neuritis. Right eye visual acuity $6 / 5$, left $6 / 9$. Left eye PVEP shows a $20 \mathrm{~ms}$ delay but the PERG shows a moderate P50 component abnormality in keeping with dysfunction distal to the optic nerve. An ophthalmological opinion was sought which suggested a resolving central serous retinopathy. In this and subsequent figures dotted vertical lines indicate the upper limit of normal latency. OD right eye, $O S$ left eye. $P V E P$ calibration $100 \mathrm{msec}, 5 \mu \mathrm{V}$. PERG calibration $50 \mathrm{msec}, 3 \mu \mathrm{V}$. 


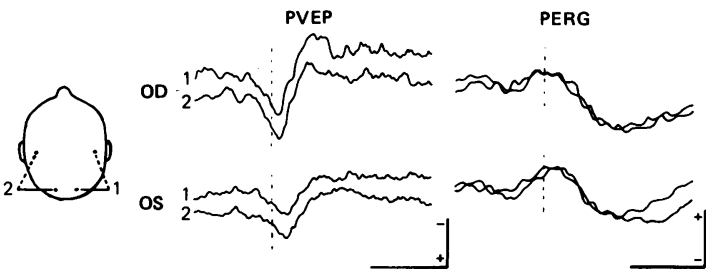

Fig 3 A 22 year old male with a four day history of bilateral blurring of vision and left retro-orbital headache was referred for neurosurgical assessment of bilateral disc swelling thought to be papilloedema. Right eye visual acuity $6 / 6$, left $6 / 18$. CT scan was normal. PVEPs are bilaterally delayed; PERGs are bilaterally delayed and reduced in keeping with probable macular dysfunction. Examination of the CSF showed 147 lymphocytes but was otherwise unremarkable. Detailed ophthalmic examination revealed bilateral serous detachments at the maculae and an overall appearance compatible with Harada's disease. $O D$ right eye, OS left eye. PVEP calibration $100 \mathrm{msec}, 5 \mu \mathrm{V}$. PERG calibration $50 \mathrm{msec}, 3 \mu \mathrm{V}$.

amplitude of $<1.0 \mu \mathrm{V}$ or an interocular ratio of $<0.30$. It is notable that PERG latency delays were not a prominent feature, only occurring in 14/84 eyes (two detachments, one central serous retinopathy, one viral retinopathy with serous detachment, one branch vein occlusion, one retinal dystrophy, one macular dystrophy, one placoid and both eyes of patients with birdshot retinopathy, Harada's disease and severe myxoedema).

No patient had a PERG abnormality confined to the N95 component. Reductions in N95 regularly occurred as would be anticipated in a component occurring subsequent to the earlier P50. The N95:P50 ratio was usually not reduced (for example figs 2 and

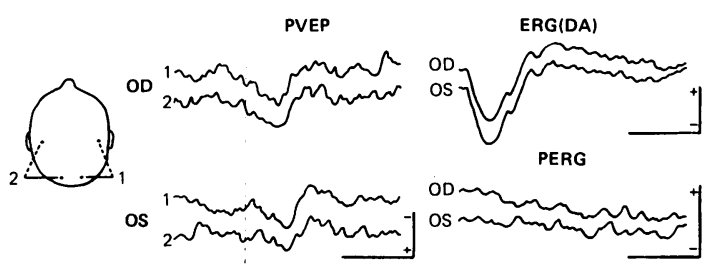

Fig 4 A 16 year old male with bilateral blurring and distortion of vision. Right eye visual acuity $6 / 12$, left $6 / 18$. The fundal appearance suggested Stargardt's disease ( $a$ macular degenerative condition) and the patient was referred for electrodiagnostic testing. The PVEPs from both eyes show a grossly abnormal P100 latency. The PERG is bilaterally extinguished in keeping with severe macular dysfunction. Flash ERGs ( 5 min dark adaptation single bright white flash) are normal. OD right eye, OS left eye. $P V E P$ calibration $100 \mathrm{msec}, 5 \mu \mathrm{V}$. ERG calibration $25 \mathrm{msec}$, $200 \mu \mathrm{V}$. PERG calibration $50 \mathrm{msec}, 2 \mu \mathrm{V}$.

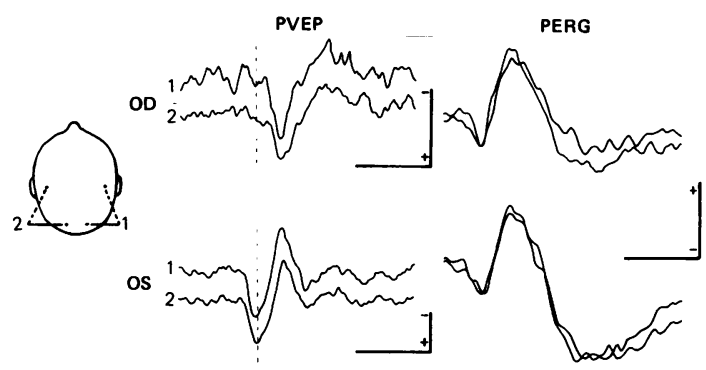

Fig 5 A 45 year old female who presented with a mid-thoracic cord lesion. Right eye visual acuity $6 / 5$, left $6 / 5$. Six years before there had been an episode of blurred vision in the right eye. Left eye findings show no definite abnormality but right eye PVEP is delayed and reduced. The PERG N95 component from the right eye shows a marked reduction; PERG P50 is normal and symmetrical. The findings are those of optic nerve dysfunction. $O D$ right eye, $O S$ left eye. PVEP calibration $100 \mathrm{msec}, 5 \mu \mathrm{V}$. PERG calibration $50 \mathrm{msec}, 2 \mu \mathrm{V}$.

3), indicating that $\mathrm{N} 95$ was not proportionally more affected than P50. Occasionally the N95:P50 ratio was reduced relative to the fellow eye but the P50 amplitude was always less than $1.0 \mu \mathrm{V}$ when thi® $\vec{\omega}$ occurred; accurate measurement of both componento may be difficult when P50 is severely abnormal. N95:P50 ratio greater than 4.0 occurred in one eys with traumatic cataract, one eye with a retinal dys 0 trophy and both eyes of a patient with birdshoo retinopathy suggesting that N95 could be relatively unaffected even though P50 was significantly reduced

\section{Discussion}

The assessment of macular dysfunction with the PERG has produced consistent findings. Abnormalities invariably occur in the main positive P50 component; ${ }^{6131516}$ there have been no previous reports of a PERG abnormality confined to the N95 component in macular disease. The results of this study are in keeping with previous reports. PVEP delays in macular dysfunction have previously been described, ${ }^{1-6}$ and these observations are also confirmed by the present study. The findings in glaucoma are less well defined. Although there are many reports of P50 component involvement, ${ }^{1317-19}$ there has been a recent report that the N95 component may be more affected. ${ }^{20}$

Initial reports of the PERG in optic nerve disease described inconsistent abnormalities of the P50 component. ${ }^{51721-24}$ Some authors concluded that the PERG was of little value in optic nerve disease. ${ }^{25}{ }^{26}$ However, it has recently been shown ${ }^{61327}$ that if the PERG is abnormal in optic nerve disease, the abnormality is usually confined to the late negative N95 component 
(fig 5). It is presumed that this reflects retrograde degeneration to the retinal ganglion cells. Many patients with optic nerve disease and delayed PVEP have a normal PERG; in our laboratory the incidence of abnormal PERG in more than 150 eyes with optic nerve demyelination is some $40 \%$ with $85 \%$ of these having an abnormality confined to the N95 component. Although a small percentage of patients with abnormal PERGs and optic nerve dysfunction show a mild P50 component reduction, there is usually a very severe PVEP abnormality and the magnitude of the reduction does not usually approach that often or present in the patients with macular dysfunction described in this report. PVEP latency delays in excess of $15 \mathrm{~ms}$ are common in optic nerve disease. It is then of particular interest that no patient in the present series with a mild PERG reduction had a PVEP delay in excess of $15 \mathrm{~ms}$; all such patients showed moderate or severe PVEP abnormalities. Acute optic neuritis may initially show a more pronounced $\mathrm{P} 50$ reduction, but in the chronic stage P50 is within the normal range and N95 reduced.$^{28}$ It should, however, be stressed that the recording of an N95 abnormality in optic nerve disease may be dependent upon the use of a high contrast stimulus (in preparation). Similarly, delays in the P50 component of the PERG are not a feature of optic nerve disease. ${ }^{6131522} 2729$

The clinical need to correctly identify the site of a PVEP delay is evident. It is the practice in some neurological units to postpone a decision on myelography in a patient with symptoms of a spinal cord lesion and possible multiple sclerosis (MS) until evoked potential recording has been performed. A delayed PVEP may lead to the cancellation of myelography by providing electrophysiological evidence of a second CNS lesion..$^{30}$ Such a patient may have a mild unilateral reduction in visual acuity or some blurring of vision. The presence of a normal PERG or a PERG abnormality confined to the $\mathrm{N} 95$ component confirms optic nerve dysfunction whereas an absent, delayed or substantially reduced PERG usually indicates more distal dysfunction as the cause of the delay.

There are often incidental findings such as amblyopia, early cataract or suboptimal refraction (the patient may simply have lost or forgotten their spectacles) in patients with suspected MS and it is important that the PVEP delays which can occur in such conditions are not mistaken for optic nerve involvement. Although ideally all patients would have their refraction checked before evoked potential recording, in practice this is seldom the case in neurological units. Equally, there is conflicting evidence for the site of dysfunction in amblyopia. Some authors suggest the retina on the basis of animal studies, ${ }^{31-33}$ while others suggest cortical dysfunction ${ }^{3435}$ or do not confirm retinal dysfunction ${ }^{36}$ and the inclusion of amblyopia in "distal anterior visual pathway dysfunction" is admittedly contentious. However, PERG P50 component reductions in amblyopia have previously been described, ${ }^{37-40}$ but not in all studies. ${ }^{41}{ }^{42}$ The present report would no longer accurately represent the routine throughput of a regional department if the patients with delayed PVEPs due to refractive error or amblyopia were excluded.

The diagnostic difficulties in swelling of the optic disc are well known. ${ }^{434}$ Optic disc swelling, although not due to raised intracranial pressure, may be referred to neurological/neurosurgical units for assessment of possible papilloedema. The initial investigation will probably be CT scanning. If no intracranial cause for raised pressure is shown then electrophysiological testing, including PERG, may help define the site of the lesion (fig 3). Papilloedema due to raised intracranial pressure does not in itself cause a PVEP abnormality. ${ }^{45}{ }^{46}$ Similarly, elderly patients in particular may have early macular changes on ophthalmoscopy which are of uncertain functional significance. We have seen one case where visual loss due to suprasellar extension of a pituitary adenoma was initially attributed to such changes. The patient suffered irreversible visual loss (NPL, 6/36) before the correct diagnosis was reached. PVEPs were extinguished but the PERG P50 component in both eyes showed no definite reduction when electrodiagnostic examination was eventually performed.

The observation that optic nerve disease could selectively affect the N95 component of the PERG ${ }^{1327}$ indicated that the PERG could assist the distinction between macular and optic nerve disease, and this was confirmed in a report which compared the findings in patients with retrobulbar neuritis to those in patients with diabetic maculopathy who also had PVEP delays. ${ }^{6}$ The present series extends these findings and further establishes the role of the PERG in the assessment of anterior visual pathway dysfunction. Knowledge of the PERG facilitates the accurate interpretation of an abnormal PVEP.

I am grateful to Dr Keith Chiappa for access to material in press; to Professor Geoffrey Arden for reading the original manuscript and to both $\mathrm{Mr}$ Chris Hogg and the Medical Photography Department, Greenwich District Hospital for assistance with the figures.

\section{References}

1 Lennerstrand G. Delayed visual evoked cortical potentials in retinal disease. Acta Ophthalmol 1982;60:497-504.

2 Bass SJ, Sherman J, Bodis-Wollner I, Nath S. Visual evoked potentials in macular disease. Invest Ophthalmol Vis Sci 1985; 26: $1071-4$ 
3 Holder GE, Chesterton JR. The visual evoked potential in Harada's disease. Neuroophthalmology 1984;4:43-5.

4 Papakostopoulos D, Dean Hart C, Cooper R, Natsikos V. Combined electrophysiological assessment of the visual system in central serous retinopathy. Electroenceph Clin Neurophysiol 1984;59:77-80.

5 Celesia GG, Kaufmann D. Pattern ERGs and visual evoked potentials in maculopathies and optic nerve diseases. Invest Ophthalmol Vis Sci 1985;26:726-35.

6 Ryan S, Arden GB. Electrophysiological discrimination between retinal and optic nerve disorders. Doc Ophthalmol 1988;68: $247-55$.

7 Holder GE. Delayed pattern VEPs: retinal or optic nerve dysfunction? Electroenceph Clin Neurophysiol 1988;69:72-3P.

8 Holder GE. The effects of chiasmal compression on the pattern visual evoked potential. Electroenceph Clin Neurophysiol 1978;45:278-80.

9 Holder GE. The visual evoked potential in ischaemic optic neuropathy. Doc Ophthalmol Proc Ser 1981;27:123-9.

10 Holder GE, Huber MJE. The effects of miosis on pattern and flash electroretinogram and pattern visual evoked potential. Doc Ophthalmol Proc Ser 1984;40:109-16.

11 Berninger TA, Schuurmans RP. Spatial tuning of the pattern ERG across temporal frequency. Doc Ophthalmol 1985;61:17-25.

12 Tan CB, King PJL, Chiappa KH. Pattern ERG: effects of reference electrode site, stimulus mode and check size. Electroenceph Clin Neurophysiol 1989;74:11-18.

13 Holder GE. Significance of abnormal pattern electroretinography in anterior visual pathway dysfunction. Br J Ophthalmol 1987; 71:166-71.

14 Holder GE. Recording the pattern electroretinogram with the Arden gold foil electrode. J Electrophysiol Technol 1988;14: 183-90.

15 Kirkham TH, Coupland SG. Abnormal pattern electroretinograms with macular cherry-red spots: evidence for selective ganglion cell damage. Curr Eye Res 1981;1:367-72.

16 Arden GB, Carter RM, MacFarlan A. Pattern and Ganzfeld electroretinograms in macular disease. Br J Ophthalmol 1984; 68:878-84.

17 Arden GB, Vaegan, Hogg CR. Clinical and experimental evidence that the pattern electroretinogram (PERG) is generated in more proximal retinal layers than the focal electroretinogram (FERG). Ann N Y Acad Sci 1982;388:214-26.

18 Wanger P, Persson HE. Pattern reversal electroretinogram in unilateral glaucoma. Invest Ophthalmol Vis Sci 1983;24:749-53.

19 Howe JW, Mitchell KW. Simultaneous recording of pattern electroretinogram and visual evoked cortical potential in a group of patients with chronic glaucoma. Doc Ophthalmol Proc Ser 1984;40:101-7.

20 Weinstein GW, Arden GB, Hitchings RA, Ryan S, Calthorpe CM, Odom JV. The pattern electroretinogram (PERG) in ocular hypertension. Arch Ophthalmol 1988;106:923-8.

21 Persson HE, Wanger P. Pattern reversal electroretinograms and visual evoked cortical potentials in multiple sclerosis. $\mathrm{Br} J$ Ophthalmol 1984;68:760-4.

22 Serra G, Carreras M, Tugnoli V, Manca M, Cristofori MC. Pattern electroretinogram in multiple sclerosis. $J$ Neurol Neurosurg Psychiatry 1984;47:879-83.

23 Boschi MC, Frosini R, Scaioli V. Correlations among clinical data, pattern electroretinogram, visual evoked potential and retinal fibre layer findings in multiple sclerosis. Doc Ophthalmol Proc Ser 1984;40:133-41.

24 Porciatti V, von Berger GP. Pattern electroretinogram and visual evoked potential in optic nerve disease: early diagnosis and prognosis. Doc Ophthalmol Proc Ser 1984;40:117-26.

25 Kirkham TH, Coupland SG. The pattern electroretinogram in optic nerve demyelination. Can J Neurol Sci 1983;10:256-60.

26 Ota I, Miyake Y. The pattern electroretinogram in patients with optic nerve disease. Doc Ophthalmol 1986;62:53-60.

27 Holder GE. Abnormalities of the pattern ERG in optic nerve $T$ lesions: changes specific for proximal retinal dysfunction. In: Barber C, Blum T, eds. Evoked Potentials III. London: $\bigcirc$ Butterworths, 1987:221-4.

28 Berninger TA, Heider W, Dodt E. Follow-up study of patients with acute optic neuritis. Invest Ophthalmol Vis Sci Suppl. . 1985;26:218.

29 Plant GT, Hess RF, Thomas SJ. The pattern evoked electroretinogram in optic neuritis: a combined psychophysical and $\bar{O}$ electrophysiological study. Brain 1986;109:469-90.

30 Mastaglia FL, Black JL, Cala LA, Collins DWK. Electro- $\overline{\bar{c}}$ physiology and avoidance of invasive neuroradiology in $\vec{\nabla}$ multiple sclerosis. Lancet 1980;i:144.

31 Ikeda H. Visual acuity, its development and amblyopia. Proc Roy Soc Med 1980;3:546-55.

32 Ikeda $\mathrm{H}$, Tremain KE. Amblyopia resulting from penalisation: $\vec{\circ}$ neurophysiological studies on kittens reared with atropinisation of one or both eyes. Br J Ophthalmol 1978;62:21-8.

33 Ikeda $\mathrm{H}$, Tremain KE. Amblyopia occurs in retinal ganglion cells in cats reared with convergent squint without alternating fixation. Exp Brain Res 1979;35:559-82.

34 Hubel DH, Wiesel TN. Effects of visual deprivation on morphology and physiology of cells in the cats lateral geniculate $N$ nucleus. $J$ Neurophysiol 1965;28:1041-59.

35 Blakemore C, van Sluyters RC. Inate and environmental factors ? in the development of the kitten cortex. $J$ Physiol 1975;248 $\vec{\omega}$ 663-716.

36 Cleland BG, Crewther DP, Crewther SG, Mitchell DE. Normalie of spatial resolution of retinal ganglion cells in cats wiţ strabismic amblyopia. J Physiol 1982;326:235-49.

37 Arden GB, Vaegan, Hogg CR, Powell DJ, Carter RM. Pattef ERGs are abnormal in many amblyopes. Trans Ophthalmol $S a$ UK 1980;100:453-60.

38 Arden GB, Wooding SL. Pattern ERG in amblyopia. Inves Ophthalmol Vis Sci 1985;26:88-96.

39 Persson HE, Wanger P. Pattern reversal electroretinograms i squint amblyopia, artificial anisometropia and simulated eccentric fixation. Acta Ophthalmol 1982;60:123-32.

40 Sokol S, Nadler D. Simultaneous electroretinograms and visually evoked potentials from adult amblyopes in response to a patterned stimulus. Invest Ophthalmol Vis Sci 1979;24:848-55.

41 Hess RF, Baker CL, Verhoeve JN, Keesey UT, France TD. The pattern evoked electroretinogram: its variability in normals and its relationship to amblyopia. Invest Ophthalmol Vis Sci 1985 26:1610-23

42 Gottlob I, Welge-Lussen L. Normal pattern electroretinograms in amblyopia. Invest Ophthalmol Vis Sci 1987;28:187-91.

43 Roper-Hall MJ. Conditions of the optic disc simulating papilloedema. In: Cant JS, ed. The Optic Nerve. London: Henry Kimpton, 1972:142-4.

44 Sanders MD. Diagnostic difficulties in optic nerve disease and in papilloedema and disc oedema. Trans Ophthalmol Soc UK 1976; 96:386-94.

45 Asselman P, Chadwick DW, Marsden CD. Visual evoked responses in the diagnosis and management of patients suspected of multiple sclerosis. Brain 1975;98:261-82.

46 Holder GE. Pattern visual evoked potential in patients with posteriorly situated space occupying lesions. Doc Ophthalmol 1985;59:121-8. 